\title{
Hedonic Rating of Sucrose Is Sub- Regionally Associated with Striatal Dopamine Transporter in Humans
}

\author{
Kyoungjune Pak ${ }^{a}$ Seongho Seo ${ }^{b}$ Myung Jun Lee ${ }^{c}$ Keunyoung Kim ${ }^{a}$ \\ Sunghwan Suh ${ }^{d}$ Jeehyun Lee ${ }^{\mathrm{e}}$ Seong-Jang Kim ${ }^{f}$ In Joo Kim ${ }^{\mathrm{a}}$
}

aDepartment of Nuclear Medicine and Biomedical Research Institute, Pusan National University Hospital, Busan, Republic of Korea; bepartment of Electronic Engineering, Pai Chai University, Daejeon, Republic of Korea; 'Department of Neurology, Pusan National University Hospital, Busan, Republic of Korea; ${ }^{\mathrm{d} D e p a r t m e n t}$ of Internal Medicine, Dong-A University College of Medicine, Busan, Republic of Korea; eDepartment of Food Science and Nutrition, Pusan National University, Busan, Republic of Korea; fDepartment of Nuclear Medicine, Pusan National University Yangsan Hospital, Yangsan, Republic of Korea

\section{Keywords}

Dopamine plasma membrane transport proteins · Feeding behavior · Sucrose · Reward

\begin{abstract}
Background: Eating behavior is determined by both homeostatic and hedonic values. Objective: We investigated the association of hedonic value with striatal dopamine transporter (DAT) availability sub-regionally. Method: An intravenous bolus injection of ${ }^{18} \mathrm{~F}-\mathrm{FP}-\mathrm{CIT}$ was administered after the infusion of glucose or placebo, and the emission data were acquired over $90 \mathrm{~min}$. DAT availability and binding potential $\left(\mathrm{BP}_{\mathrm{ND}}\right)$ were measured via the simplified reference tissue method. Subjects were assessed with sensory taste test of sucrose solutions. The "most liked" sucrose concentration (\%) was determined as the hedonic rating for sucrose. $\boldsymbol{R e}$ sults: Twenty healthy males participated in this study. After glucose loading, $\mathrm{BP}_{\mathrm{ND}} \mathrm{S}$ of putamen significantly increased, and those of caudate nucleus showed the increasing trend, while those of ventral striatum were not significantly differ-
\end{abstract}

karger@karger.com www.karger.com/nen

Karger $\stackrel{\text { ' }}{5}$

bOPEN ACCESS
(C) 2021 The Author(s)

Published by S. Karger AG, Basel

This is an Open Access article licensed under the Creative Commons Attribution-NonCommercial-4.0 International License (CC BY-NC) (http://www.karger.com/Services/OpenAccessLicense), applicable to the online version of the article only. Usage and distribution for commercial purposes requires written permission. ent. After glucose loading, the "most liked" sucrose concentration (\%) was negatively associated with $\mathrm{BP}_{\mathrm{ND}} \mathrm{S}$ of caudate nucleus and showed the trend of positive association with those from ventral striatum. Slopes of regression lines were significantly different according to the sub-regions of striatum. Conclusion: We have highlighted that striatal DAT increased after glucose loading in dorsal striatum, not in ventral striatum. These changes of striatal DAT were sub-regionally associated with the hedonic rating of sucrose from each subject.
(C) 2021 The Author(s) Published by S. Karger AG, Basel

\section{Introduction}

The brain plays a critical role in controlling the energy balance of the body [1]. Eating behavior is controlled by both the homeostatic system from hypothalamus and dorsal striatum, and the hedonic system from ventral striatum [2]. These brain circuits for the homeostatic and hedonic values are distinct, however closely linked with

Correspondence to:

Kyoungjune Pak, ilikechopin@me.com 
Fig. 1. Study design. PET, positron emission tomography

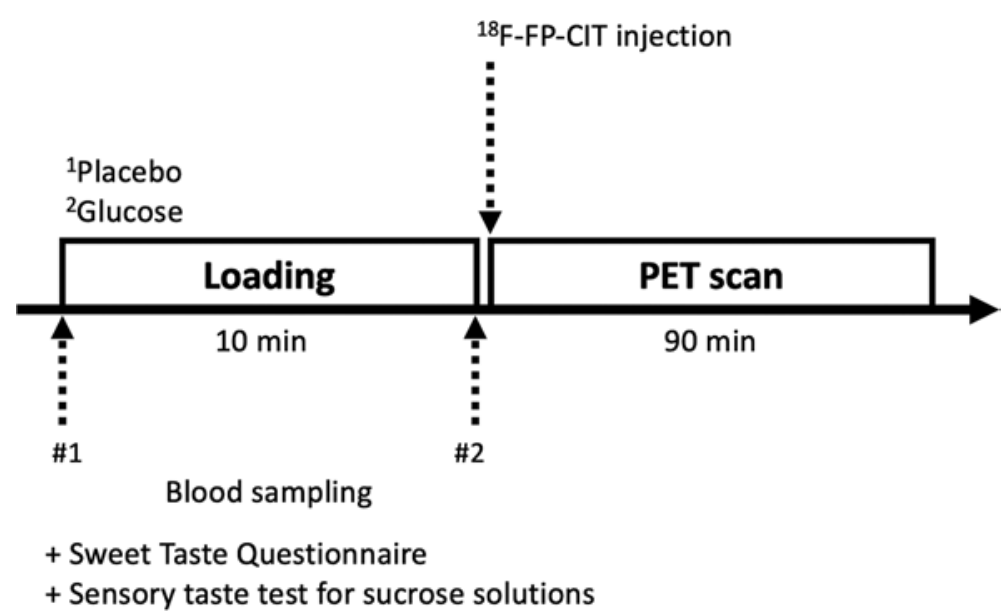

each other [3]. The decision to eat is influenced not only by the internal factor of the body, but also by external factors such as food palatability and environmental cues [4]. Dopamine is a neurotransmitter that plays a major role in the eating behavior of humans [5]. Feeding induces dopamine release in the striatum, and a dysfunction of the dopaminergic reward system can lead to overeating, which shares common underlying neurobiological mechanisms with drug addiction [6].

Brain dopamine neurotransmission is regulated by dopamine transporter (DAT), which drives reuptake of extracellular dopamine into presynaptic neurons [7]. DAT is a major target for various pharmacologically active drugs [7]. Previously, we reported the first human study of striatal DAT changes after glucose loading in healthy subjects using positron emission tomography (PET) [8]. After injection of $300 \mathrm{mg} / \mathrm{kg}$ of glucose, substantial increases of DAT availabilities were observed [8], probably by the action of insulin, the key hormone involved in the regulation of glucose metabolism [9].

Craving for sweet taste is innate and spans all ages in humans [10]. The sensitivity to mood altering effect of sweet foods and craving for sweet foods was directly associated with a higher hedonic response to sweet taste [11]. The hedonic response to sweet taste also predicted the future weight gain in humans [12]. In this study, subjects were assessed with 12-item self-reporting Sweet Taste Questionnaire (STQ) [11] and sensory taste test of sucrose solutions to evaluate subjects' response to sweet taste. Therefore, we investigated the association of hedonic rating of sucrose with striatal DAT availability subregionally as ventral striatum, caudate nucleus, and putamen.

Hedonic Rating of Sucrose and DAT

\section{Materials and Methods}

Subjects

Twenty healthy, nonobese male subjects were recruited into this study with a mean age of $23.9 \pm 1.7$ years (range $22 \sim 28$ years). Subjects who had more than $10 \%$ change in weight over 6 months, were heavy smokers, or gave history of drug abuse, brain injury, neuropsychological disorders, or endocrine disorders were excluded. All participants signed an informed consent form prior to participation. This study was approved by the institutional review board of Pusan National University Hospital. The participants in this study were included in a previous study of striatal DAT changes after glucose loading [8]. Each subject visited the institution 2 times, on separate days, for 2 PET scans with a mean interval of 9.6 days. After 2 PET scans, twenty subjects agreed on an additional visit for STQ and sensory taste test of sucrose solutions within a month. On the day of each visit, the subjects were instructed to fast overnight for at least $12 \mathrm{~h}$ and abstain from smoking and alcohol consumption. During the visits, the height (m) and weight $(\mathrm{kg})$ of the subject were measured. The BMI was calculated as follows: weight/height ${ }^{-2}$. The study design is shown in Figure 1.

\section{PET Scan}

On the day of each visit for PET scans, bilateral antecubital veins were cannulated: 1 for blood sampling and for injection of ${ }^{18} \mathrm{~F}-\mathrm{FP}-\mathrm{CIT}$, and the other for glucose or placebo infusions. The subjects were blinded and randomly assigned for either glucose or placebo infusions. Over $10 \mathrm{~min}, 300 \mathrm{mg} / \mathrm{kg}$ of glucose in a $50 \%$ solution was administered. The placebo (normal saline) was also administered in the same speed and volume [13]. The serum glucose level $(\mathrm{mg} / \mathrm{dL})$ and insulin level $(\mu \mathrm{U} / \mathrm{mL})$ were measured before and after the infusions of glucose and placebo. The serum glucose level was determined through an enzymatic reference method using hexokinase with the Glucose HK Gen.3 (Roche Diagnostics $\mathrm{GmbH}$, Mannheim, Germany). The serum insulin level was determined through an electrochemiluminescence immunoassay method using Elecsys Insulin (Roche Diagnostics GmbH, Mannheim, Germany). An intravenous bolus injection of ${ }^{18} \mathrm{~F}$-FP- 
Fig. 2. The paired $t$ test of $\mathrm{BP}_{\mathrm{ND}}$ of ventral striatum (a), caudate nucleus (b), and putamen (c) between glucose loading and placebo loading. $\mathrm{BP}_{\mathrm{ND}}$, binding potential.

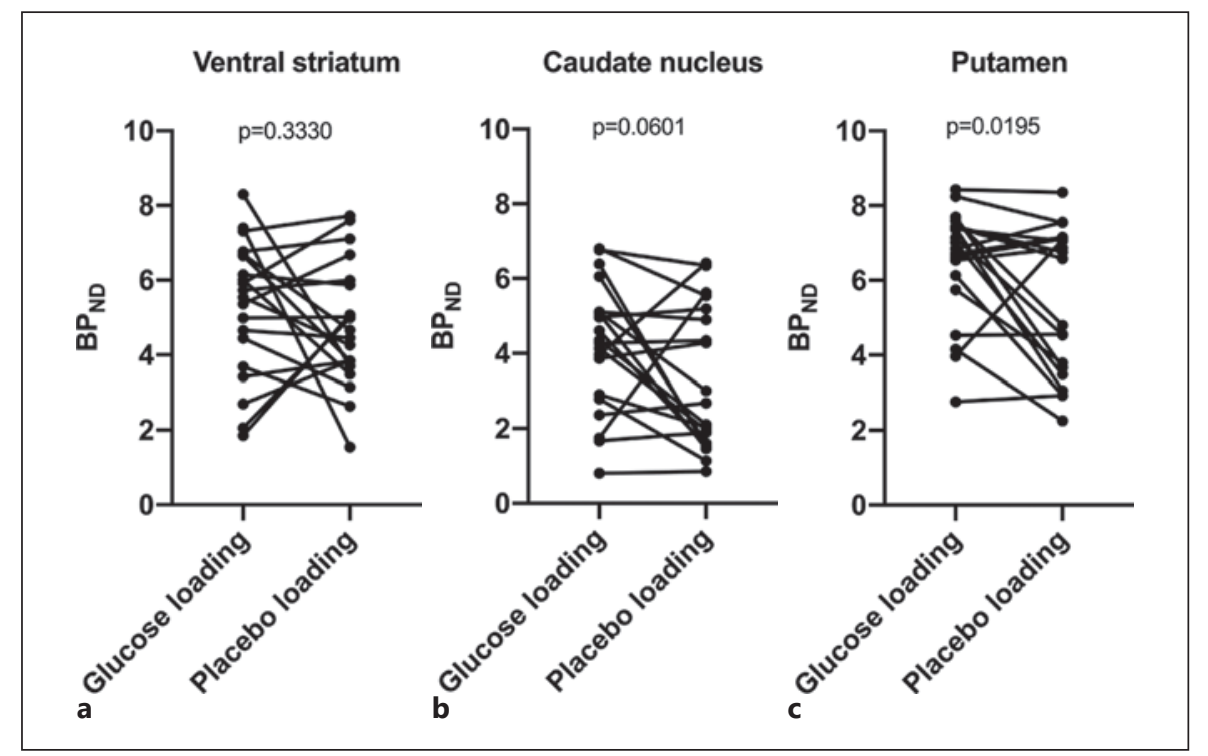

CIT $(210.9 \pm 16.3 \mathrm{MBq})$ was administered after the infusion of glucose or placebo. The emission data were acquired over $90 \mathrm{~min}$ with 50 frames of progressively increasing durations $(15 \mathrm{~s} \times 8$ frames, $30 \mathrm{~s} \times 16$ frames, $60 \mathrm{~s} \times 10$ frames, $240 \mathrm{~s} \times 10$ frames, and $300 \mathrm{~s} \times 6$ frames) using the Siemens Biograph 40 Truepoint PET/ CT (Siemens Healthcare, Knoxville, TN, USA). The dynamic PET data were collected in the 3-dimensional mode, with 148 slices with image sizes of $256 \times 256$ and pixel sizes of $1.3364 \times 1.3364 \mathrm{~mm}^{2}$. These were reconstructed using an iterative method with a Gaussian filter.

\section{Image Analysis}

For a volume-of-interest (VOI)-based analysis, an averaged image (0-10 min after injection) was created from dynamic PET frames and spatially normalized to ${ }^{15} \mathrm{O}$-water PET template in statistical parametric mapping 5 (Wellcome Trust Centre for Neuroimaging, London, UK). To extract time-activity curves of VOIs from full dynamic PET scans, Oxford-GSK-Imanova striatal atlas from FMRIB Software Library v. 5.0 (https://fsl.fmrib.ox.ac.uk/ fsl) was applied, which is an atlas involving sub-striatal regions of ventral striatum, caudate nucleus, and putamen segmented according to the anatomical structure, and manually delineated on the nonlinear MNI 152 template [14]. DAT availability, expressed in terms of binding potential $\left(\mathrm{BP}_{\mathrm{ND}}\right)$, was measured by analyzing time-activity curves via the simplified reference tissue method [15] with a reference of cerebellum. Image analysis was done using PMOD version 3.6 (PMOD Technologies LLC, Zurich, Switzerland).

For a voxel-based analysis, parametric maps were generated for each subject and analyzed for regression with the "most liked" sucrose concentration (\%). Results were displayed at a significance threshold of uncorrected $p<0.005$ and minimum cluster size for 10 contiguous voxels. Image analysis was done using SPM8 (Wellcome Trust Centre for Neuroimaging, London, UK) implemented on MATLAB R2013a (MathWorks, MA, USA).
Sweet Taste Questionnaire and Sensory Taste Test of Sucrose Solutions

On the day of the 3rd visit, subjects were assessed with 12-item self-reporting STQ to evaluate subjects' reactions to eating sweets, craving for sweets, and degree of control over eating sweet foods. There were 2 subscales in STQ: STQ1, mood altering effect of sweet food and STQ2, impaired control over eating sweets [11]. After completion of STQ, subjects received sucrose (Sucrose, Alfa Aesar, MA, USA) in solution $(2,4,6,8,10,12,14$, and $16 \%)$. They were instructed to taste each concentration of sucrose solution and scored it for sweetness using the 9-point hedonic scale that they were given as verbal scale ranging from dislike extremely to like extremely. Sucrose solutions were labeled with 3-digit random numbers, and the order of tasting was randomized. The "most liked" sucrose concentration (\%) was determined as the hedonic scale of each subject for sucrose. Average concentration was used when there were more than 1 concentration levels with the same liking score.

\section{Statistical Analysis}

Normality was assessed using the D'Agostino \& Pearson normality test. The one-way analysis of variance was used to test the difference of the hedonic scales for sucrose between concentrations of sucrose solution. Percentage changes of $\mathrm{BP}_{\mathrm{ND}}$ were calculated as follows: $\left(\mathrm{BP}_{\mathrm{ND}}\right.$ after glucose loading $-\mathrm{BP}_{\mathrm{ND}}$ after placebo loading $) / \mathrm{BP}_{\mathrm{ND}}$ after placebo loading $\times 100(\%)$. The paired $t$ test was used to compare $\mathrm{BP}_{\mathrm{ND}} \mathrm{S}$ after glucose loading and those after placebo loading from each subject. The Pearson correlation analysis was used to determine whether the variables (the "most liked" sucrose concentration, $\mathrm{STQ}, \mathrm{BMI}$, and $\mathrm{BP}_{\mathrm{ND}}$ ) were associated. The linear regression analysis was used to model the relationship between the "most liked" sucrose concentration (\%) and BP $\mathrm{ND}_{\mathrm{ND}}$. Analysis of covariance was used to compare slopes of linear regression lines between the "most liked" sucrose concentration and $\mathrm{BP}_{\mathrm{ND}}$ according to sub-regions of striatum (ventral striatum, caudate nucleus, and putamen). All analyses were conducted using Prism (v7.0d, GraphPad Software Inc, La Jolla, CA, USA). 


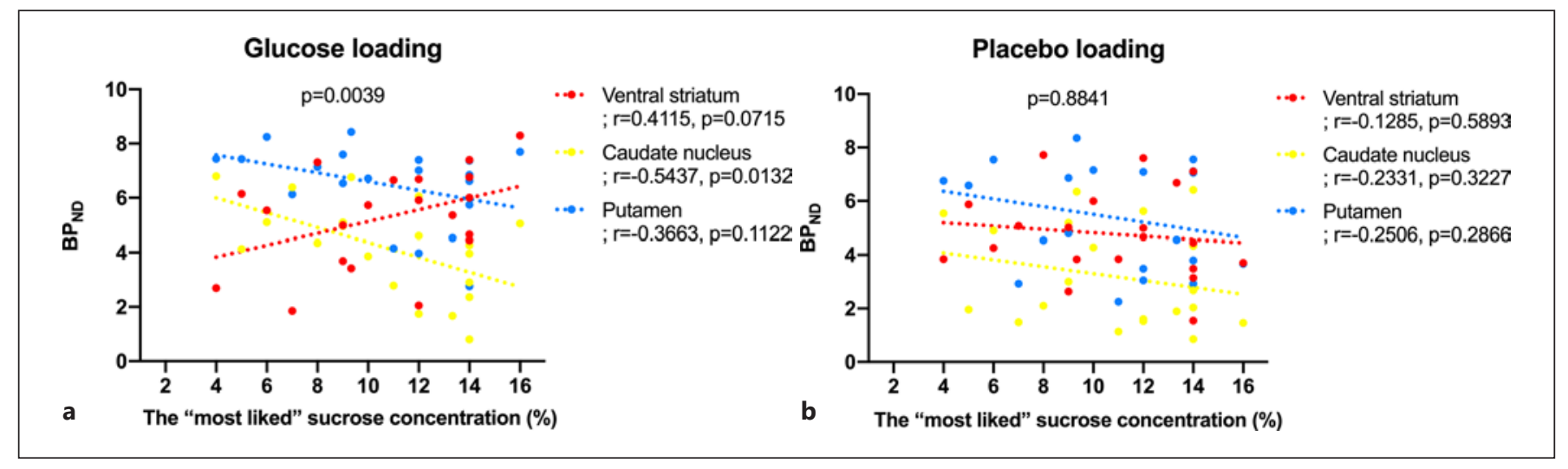

Fig. 3. Regression analysis between the "most liked" sucrose concentration (\%) and $\mathrm{BP}_{\mathrm{ND}}$ after glucose loading (a) and placebo loading (b). $\mathrm{BP}_{\mathrm{ND}}$, binding potential.

\section{Results}

Twenty healthy males were participated in this study. The mean BMI of the study group was $22.9 \mathrm{~kg} / \mathrm{m}^{2}$ ranging between 18.9 and $26.4 \mathrm{~kg} / \mathrm{m}^{2}$. After glucose loading, the glucose (mean $21 \mathrm{mg} / \mathrm{dL}$ ) and the insulin (mean $7 \mu \mathrm{U} /$ $\mathrm{mL}$ ) levels were increased. The $12 \%$ sucrose solution received the highest liking score, however was not significantly different from $10 \%$. BMI was associated neither with the "most liked" sucrose concentration $(r=-0.1812$; $p=0.4446)$, nor with STQ (STQ1; $r=-0.1525, p=0.5210 /$ STQ2; $r=0.0266, p=0.9115)$. The "most liked" sucrose concentration (\%) showed the trend toward the positive correlation with STQ1 $(r=0.3962, p=0.0837)$, not with STQ2 $(r=-0.0376, p=0.8750)$.

After glucose loading, $\mathrm{BP}_{\mathrm{ND}} \mathrm{S}$ of putamen were significantly increased ( $p=0.0195$, mean percentage change of $\mathrm{BP}_{\mathrm{ND}} \pm$ standard deviation; $\left.+33.7 \pm 51.7 \%\right)$, and those of caudate nucleus showed the increasing trend $(p=0.0601$, $+70.4 \pm 113.6 \%$ ), while those of ventral striatum were not significantly different $(p=0.3330,+29.6 \pm 94.0 \%)$ compared with those after placebo loading (Fig. 2). The relationships between the "most liked" sucrose concentration (\%) and $\mathrm{BP}_{\mathrm{ND}} \mathrm{S}$ were investigated by both VOI-based and voxel-based analyses. With a VOI-based analysis, after glucose loading, the "most liked" sucrose concentration (\%) was negatively associated with $\mathrm{BP}_{\mathrm{ND}}$ s of caudate nucleus ( $r=-0.5437, p=0.0132)$ and showed the trend of positive association with those from ventral striatum $(r=$ $0.4115, p=0.0715)$. Slopes of regression lines were significantly different according to the sub-regions of striatum $(F=6.150, p=0.0039)$ (Fig. 3a). After placebo loading, the "most liked" sucrose concentration (\%) was not associated with $\mathrm{BP}_{\mathrm{ND}} \mathrm{S}$ of ventral striatum $(r=-0.1285, p$
$=0.5893)$, caudate nucleus $(r=-0.2331, p=0.3227)$, and putamen $(r=-0.2506, p=0.2866)$ without the significant difference in slopes of regression lines $(F=0.1235, p=$ 0.8841 ) (Fig. 3b). With a voxel-based analysis, the "most liked" sucrose concentration (\%) was not associated with DAT availabilities of brain regions except for left putamen (MNI coordinates; $-24,12,8, k=87, T=3.51$ ) and left caudate nucleus (MNI coordinates; $-18,14,12 ;-16$, $-6,24, k=104, T=3.36 ; 3.07)$ negatively after glucose loading (Fig. 4).

\section{Discussion}

The results of the current study indicate the following. First, DAT availabilities after glucose loading were increased in dorsal striatum (caudate nucleus and putamen), not in ventral striatum. Second, DAT availabilities after glucose loading were sub-regionally related to the "most liked" sucrose concentration for sucrose.

Previously, we reported the first human study of striatal DAT changes after glucose loading in healthy subjects [8]. After injection of $300 \mathrm{mg} / \mathrm{kg}$ of glucose, substantial increases of DAT availabilities were observed [8]. This might have been done by the action of insulin, the key hormone involved in the regulation of glucose metabolism [9]. Insulin is released from the beta cells of the pancreas in response to increased glucose levels as a consequence of food intake [9]. After crossing the blood-brain barrier, insulin activates the PI3K/Akt signaling pathway by acting on insulin receptors, which enhances surface expression of DAT [16]. DAT, located on the plasma membrane [17], drives reuptake of extracellular dopamine into presynaptic neurons [7]. As DATs take up the 


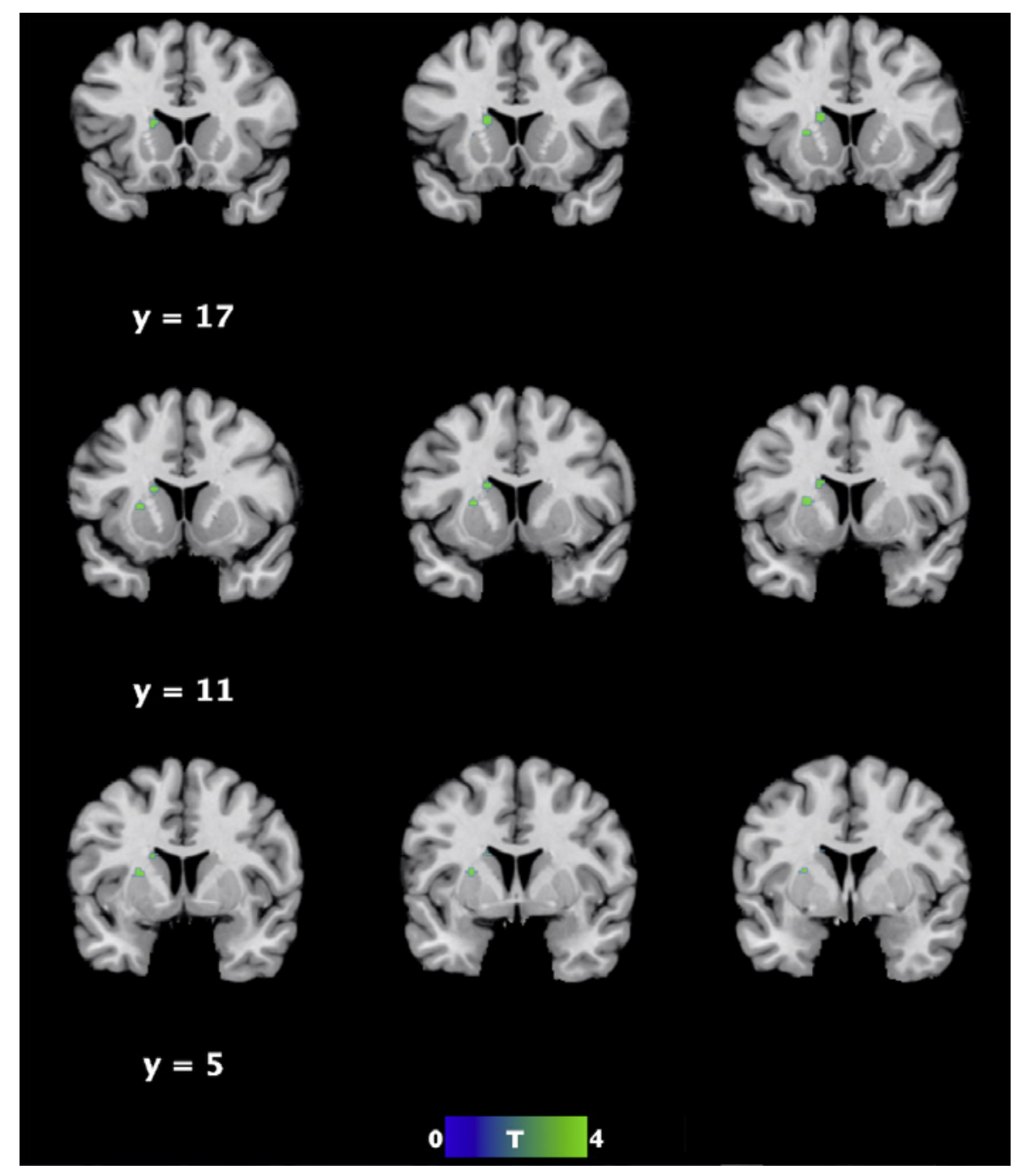

Fig. 4. Negatively associated brain regions with the "most liked" sucrose concentration (\%) after glucose loading: left putamen (MNI coordinates; $-24,12,8, k=87, T=$ 3.51) and left caudate nucleus (MNI coordinates; $-18,14,12 ;-16,-6,24, k=104$, $T=3.36 ; 3.07)$.

synaptic dopamine into the presynaptic neuron, higher expression of striatal DAT might result in a higher clearance of synaptic dopamine leading to lower concentrations of synaptic dopamine, which might stop food intake. In addition, DAT is a major target for various pharmacologically active drugs, or substances, such as cocaine and amphetamine [7].

Dopamine receptor has been investigated widely in the field of neuroimaging with regard to eating behavior and obesity. However, there is a fundamental limitation of dopamine receptor availability acquired by PET scans, as endogenous dopamine competes with the radiopharmaceuticals for binding with dopamine receptor [18]. Therefore, a lower dopamine receptor availability can be interpreted as either a higher release of endogenous dopamine or a downregulation of dopamine receptors [18]. Different from dopamine receptor, DAT availability acquired by PET scans is not affected by endogenous dopamine concentrations $[19,20]$. However, DAT was not supposed to be involved in the neurobiology of eating behavior in humans, as DAT availability was not correlated with BMI in previous studies [21-23].

Eating behavior is thought to be determined by both homeostatic and hedonic values [24]. Homeostatic value is related to maintaining the body weight, balancing of energy, and nutrients [24]. Hypothalamus expresses receptors for neuropeptides associated with homeostatic feeding [25]. Also, dorsal striatum involves in regulating eating behavior by maintaining caloric requirements for survival [26]. With ${ }^{11} \mathrm{C}$-raclopride PET, Volkow et al. [26] demonstrated that dorsal striatum might be involved in nonhedonic food motivation. On the other hand, hedonic value is linked to sensory perception or pleasure after eating behavior tied to memories, emotions, and events 
$[27,28]$. The dopamine pathway projecting from the ventral tegmental area to ventral striatum (including nucleus accumbens) plays a major role in processing reward cues and motivation to seek palatable foods [29]. Abnormalities that change the setting of energetic balance or the sensitivity to food rewards might lead to food addiction, inability to control appetite, or obesity [4]. Separate brain circuits for the homeostatic and hedonic values are known to mediate eating behavior [2]. In mice, during sugar intake, suppressing hedonic value inhibited dopamine release in ventral striatum, not in the dorsal striatum [2]. On the contrary, suppressing nutritional value inhibited dopamine release in dorsal, not in ventral striatum [2]. However, these brain circuits are not completely dissociable from 1 another with overlapping features, as both of them are activated during eating behavior [28]. The degree of activation of each brain circuit may be dependent on the type of food and the physiological state of humans [28]. In this study, DAT availabilities were measured after intravenous glucose loading. Glucose loading increases the blood glucose level with an effect on homeostatic value of the body without adding hedonic value. In this regard, insulin released from the pancreas in response to increased glucose levels might act on dorsal striatum resulting in the increases of DAT availabilities. However, DAT availabilities of ventral striatum showed the different responses to glucose loading. After glucose loading, DAT availabilities of ventral striatum did not increase or decrease, however showed the trend of positive association with the "most liked" sucrose concentration, and the regression line of ventral striatum was significantly different from those of dorsal striatum. As hedonic response to sweet taste is known to be a stable trait [30] and closely linked to the perception of taste [31], DAT of ventral striatum may react to insulin according to hedonic value of each subject. DAT availability is not affected by endogenous dopamine concentrations [19,20]; therefore, the changes of DAT availabilities after glucose loading might reflect the direct response to increased blood glucose level, a change in homeostatic factor of eating behavior. Previously, Pepino et al. [10] reported the association between sucrose preference and dopamine receptor availability from ${ }^{11} \mathrm{C}-\mathrm{NMB}$ PET scans. Preferences for higher sucrose concentration were correlated with lower dopamine receptor availabilities from both ventral and dorsal striata in nonobese subjects [10]. However, this was not observed in obese subjects, probably due to the disruption of dopaminergic neural circuits [10].

There are several limitations to this study. First, twenty healthy males were included; as such, the sample size was small. Second, as nonobese subjects without endocrine disorders were included in this study, we could not investigate the effect of obesity on the hedonic response to sweet taste. Third, $\mathrm{BP}_{\mathrm{ND}} \mathrm{s}$ that were measured did not distinguish between DAT density and affinity.

We have highlighted that striatal DAT increased after glucose loading in dorsal striatum, not in ventral striatum. These changes of striatal DAT were sub-regionally associated with the hedonic rating of sucrose from each subject.

\section{Statement of Ethics}

Ethics approval was done from institutional review board $(\mathrm{Pu}-$ san National University Hospital: PNUH-1707-019-057). Written informed consent was obtained from all subjects enrolled in this study.

\section{Conflict of Interest Statement}

The authors had no competing interests.

\section{Funding Sources}

This research was supported by Basic Science Research Program through the National Research Foundation of Korea (2020R1F1A1054201) and Clinical research grant from Pusan National University Hospital in 2021.

\section{Author Contributions}

Kyoungjune Pak: study design, wrote the manuscript, and statistical analysis; Seongho Seo and Myung Jun Lee: study design and wrote the manuscript; Sunghwan Suh, Jeehyun Lee, and Keunyoung Kim: study design; Seong-Jang Kim and In Joo Kim: study design and statistical analysis.

\section{Availability of Data and Material}

The data that support the findings of this study are available on request from the corresponding author.

\begin{tabular}{|c|c|}
\hline References & $\begin{array}{l}1 \text { Morton GJ, Meek TH, Schwartz MW. Neuro- } \\
\text { biology of food intake in health and disease. } \\
\text { Nat Rev Neurosci. } 2014 \text { Jun;15(6):367-78. } \\
2 \text { Tellez LA, Han W, Zhang X, Ferreira TL, Per- } \\
\text { ez IO, Shammah-Lagnado SJ, et al. Separate } \\
\text { circuitries encode the hedonic and nutritional } \\
\text { values of sugar. Nat Neurosci. } 2016 \text { Mar; } \\
\text { 19(3):465-70. } \\
3 \text { van Galen KA, Ter Horst KW, Booij J, la Fleur } \\
\text { SE, Serlie MJ. The role of central dopamine } \\
\text { and serotonin in human obesity: lessons } \\
\text { learned from molecular neuroimaging stud- } \\
\text { ies. Metabolism. } 2018 \text { Aug;85:325-39. }\end{array}$ \\
\hline
\end{tabular}


4 Volkow ND, Wang GJ, Tomasi D, Baler RD. The addictive dimensionality of obesity. Biol Psychiatry. 2013 May 1;73(9):811-8.

5 Baik JH. Dopamine signaling in reward-related behaviors. Front Neural Circuits. 2013;7: 152.

6 Volkow ND, Wang GJ, Baler RD. Reward, dopamine and the control of food intake: implications for obesity. Trends Cogn Sci. 2011 Jan;15(1):37-46.

7 Vaughan RA, Foster JD. Mechanisms of dopamine transporter regulation in normal and disease states. Trends Pharmacol Sci. 2013 Sep;34(9):489-96.

8 Pak K, Seo S, Kim K, Lee MJ, Shin MJ, Suh S, et al. Striatal dopamine transporter changes after glucose loading in humans. Diabetes Obes Metab. 2020 Jan;22(1):116-22.

9 Schwartz MW, Woods SC, Porte DJr, Seeley RJ, Baskin DG. Central nervous system control of food intake. Nature. 2000 Apr 6; 404(6778):661-71.

10 Pepino MY, Eisenstein SA, Bischoff AN, Klein S, Moerlein SM, Perlmutter JS, et al. Sweet dopamine: sucrose preferences relate differentially to striatal D2 receptor binding and age in obesity. Diabetes. 2016 Sep;65(9): 2618-23.

11 Kampov-Polevoy AB, Alterman A, Khalitov E, Garbutt JC. Sweet preference predicts mood altering effect of and impaired control over eating sweet foods. Eat Behav. 2006 Aug; 7(3):181-7.

12 Salbe AD, DelParigi A, Pratley RE, Drewnowski A, Tataranni PA. Taste preferences and body weight changes in an obesity-prone population. Am J Clin Nutr. 2004 Mar;79(3): $372-8$.
13 Haltia LT, Rinne JO, Merisaari H, Maguire RP, Savontaus E, Helin S, et al. Effects of intravenous glucose on dopaminergic function in the human brain in vivo. Synapse. 2007 Sep;61(9):748-56.

14 Tziortzi AC, Searle GE, Tzimopoulou S, Salinas $C$, Beaver JD, Jenkinson $M$, et al. Imaging dopamine receptors in humans with [11C](+)-PHNO: dissection of D3 signal and anatomy. Neuroimage. 2011 Jan 1;54(1):264-77.

15 Lammertsma AA, Hume SP. Simplified reference tissue model for PET receptor studies. Neuroimage. 1996 Dec;4(3 Pt 1):153-8.

16 Jones KT, Woods C, Zhen J, Antonio T, Carr $\mathrm{KD}$, Reith ME. Effects of diet and insulin on dopamine transporter activity and expression in rat caudate-putamen, nucleus accumbens, and midbrain. J Neurochem. 2017 Mar; 140(5):728-40.

17 Chen N, Reith ME. Structure and function of the dopamine transporter. Eur J Pharmacol. 2000 Sep 29;405(1-3):329-39.

18 Guo J, Simmons WK, Herscovitch P, Martin A, Hall KD. Striatal dopamine D2-like receptor correlation patterns with human obesity and opportunistic eating behavior. Mol Psychiatry. 2014 Oct;19(10):1078-84.

19 Kilbourn MR, Butch ER, Desmond T, Sherman P, Harris PE, Frey KA. In vivo [11C]dihydrotetrabenazine binding in rat striatum: sensitivity to dopamine concentrations. Nucl Med Biol. 2010 Jan;37(1):3-8.

20 Booij J, van Loon G, de Bruin K, Voorn P. Acute administration of haloperidol does not influence 123I-FP-CIT binding to the dopamine transporter. J Nucl Med. 2014 Apr; 55(4):647-9.

21 Thomsen G, Ziebell M, Jensen PS, da CuhnaBang S, Knudsen GM, Pinborg LH. No correlation between body mass index and striatal dopamine transporter availability in healthy volunteers using SPECT and [123I]PE2I. Obesity. 2013 Sep;21(9):1803-6.
22 van de Giessen E, Hesse S, Caan MW, Zientek F, Dickson JC, Tossici-Bolt L, et al. No association between striatal dopamine transporter binding and body mass index: a multi-center European study in healthy volunteers. Neuroimage. 2013 Jan 1;64:61-7.

23 Nam SB, Kim K, Kim BS, Im HJ, Lee SH, Kim SJ, et al. The effect of obesity on the availabilities of dopamine and serotonin transporters. Sci Rep. 2018 Mar 21;8(1):4924.

24 Volkow ND, Wang GJ, Maynard L, Jayne M, Fowler JS, Zhu W, et al. Brain dopamine is associated with eating behaviors in humans. Int J Eat Disord. 2003 Mar;33(2):136-42.

25 Cone RD. Anatomy and regulation of the central melanocortin system. Nat Neurosci. 2005 May;8(5):571-8.

26 Volkow ND, Wang GJ, Fowler JS, Logan J, Jayne M, Franceschi D, et al. "Nonhedonic" food motivation in humans involves dopamine in the dorsal striatum and methylphenidate amplifies this effect. Synapse. 2002 Jun 1; 44(3):175-80.

27 Michaelides M, Thanos PK, Volkow ND, Wang GJ. Translational neuroimaging in drug addiction and obesity. ILAR J. 2012; 53(1):59-68.

28 Rossi MA, Stuber GD. Overlapping brain circuits for homeostatic and hedonic feeding. Cell Metab. 2018 Jan 9;27(1):42-56.

29 Caravaggio F, Raitsin S, Gerretsen P, Nakajima S, Wilson A, Graff-Guerrero A. Ventral striatum binding of a dopamine D2/3 receptor agonist but not antagonist predicts normal body mass index. Biol Psychiatry. 2015 Jan 15; 77(2):196-202.

30 Looy H, Weingarten HP. Effects of metabolic state on sweet taste reactivity in humans depend on underlying hedonic response profile. Chem Senses. 1991;16(2):123-30.

31 Drewnowski A. Taste preferences and food intake. Annu Rev Nutr. 1997;17:237-53. 